\title{
Bioguided Isolation, Characterization, and Biotransformation by Fusarium verticillioides of Maize Kernel Compounds That Inhibit Fumonisin Production
}

\author{
Vessela Atanasova-Penichon, ${ }^{1}$ Stéphane Bernillon, ${ }^{2,3}$ Gisèle Marchegay, ${ }^{1}$ Aurélia Lornac, ${ }^{1}$ Laetitia \\ Pinson-Gadais, ${ }^{1}$ Nadia Ponts, ${ }^{1}$ Enric Zehraoui, ${ }^{1}$ Christian Barreau, ${ }^{4}$ and Florence Richard-Forget ${ }^{1}$ \\ ${ }^{1}$ INRA, UR1264 MycSA; ${ }^{2}$ INRA, UMR1332 Biologie du Fruit et Pathologie; ${ }^{3}$ Metabolome Facility of Bordeaux Functional \\ Genomics Center, IBVM; and ${ }^{4}$ CNRS, UR1264 MycSA, 71 Avenue Edouard Bourlaux, CS 20032, 33882 Villenave d'Ornon \\ cedex, France
}

Submitted 29 January 2014. Accepted 1 July 2014.

Fusarium verticillioides infects maize ears, causing ear rot disease and contamination of grain with fumonisin mycotoxins. This contamination can be reduced by the presence of bioactive compounds in kernels that are able to inhibit fumonisin biosynthesis. To identify such compounds, we used kernels from a maize genotype with moderate susceptibility to $F$. verticillioides, harvested at the milk-dough stage (i.e., when fumonisin production initiates in planta), and applied a bioguided fractionation approach. Chlorogenic acid was the most abundant compound in the purified active fraction and its contribution to fumonisin inhibitory activity was up to $70 \%$. Moreover, using a set of maize genotypes with different levels of susceptibility, chlorogenic acid was shown to be significantly higher in immature kernels of the moderately susceptible group. Altogether, our data indicate that chlorogenic acid may considerably contribute to either maize resistance to Fusarium ear rot, fumonisin accumulation, or both. We further investigated the mechanisms involved in the inhibition of fumonisin production by chlorogenic acid and one of its hydrolyzed products, caffeic acid, by following their metabolic fate in supplemented $F$. verticillioides broths. Our data indicate that $F$. verticillioides was able to biotransform these phenolic compounds and that the resulting products can contribute to their inhibitory activity.

Fusarium ear rot, which is caused by Fusarium proliferatum, F. verticillioides, and related species such as F. subglutinans, is one of the most important diseases that affect corn production. F. verticillioides and $F$. proliferatum are also considered to be major sources of contamination with a wide range of mycotoxins that include fusaric acid, fusarins, and fumonisins (Brown et al. 2012). Fumonisins, a family of

Corresponding author: V. Atanasova-Penichon; E-mail: vessela.atanasovapenichon@bordeaux.inra.fr

* The $\boldsymbol{e}$-Xtra logo stands for "electronic extra" and indicates that a supplementary figure is published online.

This article is in the public domain and not copyrightable. It may be freely reprinted with customary crediting of the source. The American Phytopathological Society, 2014. polyketide-derived mycotoxins, are the most prevalent and have been associated with esophageal cancer in humans, leukoencephalomalacia in horses, pulmonary edema in pigs, and cancer-promoting activity in rats (Gelderblom et al. 2004; Voss et al. 2002). Among fumonisins, fumonisin B1 (FB1), FB2, and $\mathrm{FB} 3$ are the most frequently encountered in maize kernels, FB1 occurring at the highest concentration. Toxin levels in food and feed have been subjected to European regulations, limiting the FB1 + FB2 content in unprocessed maize for human consumption to a maximum level of $4 \mathrm{mg} / \mathrm{kg}$ (Commission regulation [EC] number 1126/2007). Fumonisins are heat-stable molecules that are not fully eliminated during food processing (Humpf and Voss 2004). Thus, the best way to reduce or prevent contamination is to control fumonisin biosynthesis at the field level during plant cultivation. The three major factors influencing fungal development and mycotoxin production in kernels are environmental conditions, agricultural practices, and susceptibility range of genotypes (Edwards 2004). Increasing efforts in the corn seed industry started with the identification of genetic sources for maize Fusarium ear rot resistance. Several approaches are currently under investigation. Among them, the identification of naturally occurring mechanisms in plants that lead to reduced mycotoxin accumulation seems particularly relevant. One of these mechanisms includes mycotoxin detoxification by metabolic transformation (e.g., glucosylation or acetylation) of the toxin (Berthiller et al. 2009). Others result from the occurrence of endogenous plant compounds, either constitutive or induced in response to pathogen infection, that can inhibit toxin biosynthesis (Boutigny et al. 2008). In addition to temperature, water availability, $\mathrm{pH}$, and nutrient sources, plant-produced secondary metabolites are factors of the $F$. verticillioides-maize environment that can eventually counteract fumonisin production (Picot et al. 2010). Among these, phenolic compounds have been the subject of intensive research and characterized for their in vitro efficiency to inhibit the productions of various mycotoxins, including fumonisins (Beekrum et al. 2003; Dambolena et al. 2008; Samapundo et al. 2007), type B trichothecenes, (Boutigny et al. 2009), and aflatoxins (Chipley and Uraih 1980; Norton 1999). In addition to phenolic compounds, plant tocopherols and carotenoids were recently characterized for their effect on fumonisin production (Picot et al. 2013). Sublethal doses of $\alpha-$ tocopherol were shown to significantly affect fumonisin yield, whereas no effect was ascribed to concentrations of carotenoids, 
consistent with physiological levels determined in maize kernels (Picot et al. 2013).

Additional information on metabolic factors able to interfere with fumonisin accumulation is expected with the development of metabolomic approaches. Indeed, regarding type B trichothecene contamination, metabolomic analysis aiming at comparing metabolite abundance in mature kernels of $F$. graminearumresistant versus -susceptible genotypes of barley and wheat led to the identification of candidate compounds belonging to four main metabolic pathways: fatty acid, phenylpropanoid, flavonoid, and terpenoid (Bollina et al. 2010; Kumaraswamy et al. 2011). A similar but more restricted approach was recently applied to the pathosystem $F$. verticillioides-maize (CamposBermudez et al. 2013). Metabolomic profile of resistant and susceptible maize kernels was analyzed and the potential role of polyamines highlighted. Therefore, several constitutive or resistant-metabolites potentially able to limit fumonisin accumulation in maize kernels have been identified. However, their respective importance remains to be clarified. Moreover, the mechanisms by which plant metabolites interfere with fumonisin accumulation have not been clearly described, including the mechanisms by which these compounds could be biotransformed by $F$. verticillioides. Indeed, apart from their inhibitory efficiency, the potential key role of such compounds is directly linked to their abundance and bioavailability for $F$. verticillioides at the time fumonisin production occurs in developing maize kernels. In a previous field study, fumonisin production was found to be initiated on immature maize kernels at the milkdough stage (Picot et al. 2011).

The aim of this work is to identify the compounds found in developing maize kernels that contribute most of the resistance to fumonisin accumulation. A bioguided fractionation strategy was applied to a methanolic extract of maize kernels harvested at the milk-dough stage that shows a significant fumonisin capacity to inhibit fumonisin production (inhibitory activity). The identified active compounds were analyzed for their efficiency in inhibiting fumonisin production together with the mechanisms by which they can be biotransformed by $F$. verticillioides. Using a panel of maize genotypes, we further investigated the relationship between maize resistance level to Fusarium ear rot and abundance of the previously identified active compound in early stages of kernel development.

\section{RESULTS}

\section{Bioactive compounds from a maize kernel extract with significant fumonisin inhibitory activity.}

Kernels of the maize inbred line with moderate susceptibility to $F$. verticillioides were harvested 20 days after silking, ground, lyophilized, and extracted with hexane (nonpolar compounds), methanol/ $\mathrm{H}_{2} \mathrm{O}$ (semipolar compounds), or $\mathrm{H}_{2} \mathrm{O}$ (polar compounds). The three extracts were compared in their ability to affect fungal growth and fumonisin biosynthesis by Fv 63 . The methanol $/ \mathrm{H}_{2} \mathrm{O}$ extract was the only one that induced a significant inhibition of fumonisin yield (data not shown). At the concentration used $(5 \mathrm{mg}$ of lyophilized extract in $10 \mathrm{ml}$ of liquid medium), fungal growth was not affected. The methanol/ $\mathrm{H}_{2} \mathrm{O}$ extract was filtered, concentrated, and then fractionated by size-exclusion chromatography using a Toyopearl gel with exclusion limits between 100 and 10,000 Da. Absorbances were monitored at 230, 280, and $320 \mathrm{~nm}$ and four pools of fractions were defined accordingly and labeled F1, F2, F3, and F4, as shown on the resulting chromatogram reported in Figure 1A. The four fractions were tested for their ability to affect Fv 63 growth and fumonisin production. None of the fractions exhibited an antifungal activity and fumonisin inhibitory compounds were eluted in the F2 fraction (Fig. 1B). The F2 fraction reduced fumonisin production by $65 \%$ compared with the corresponding control $\mathrm{C} 1$. Composition of the F2 fraction was analyzed using liquid chromatography diode array detection (LC/DAD), and the obtained chromatograms at 260 and
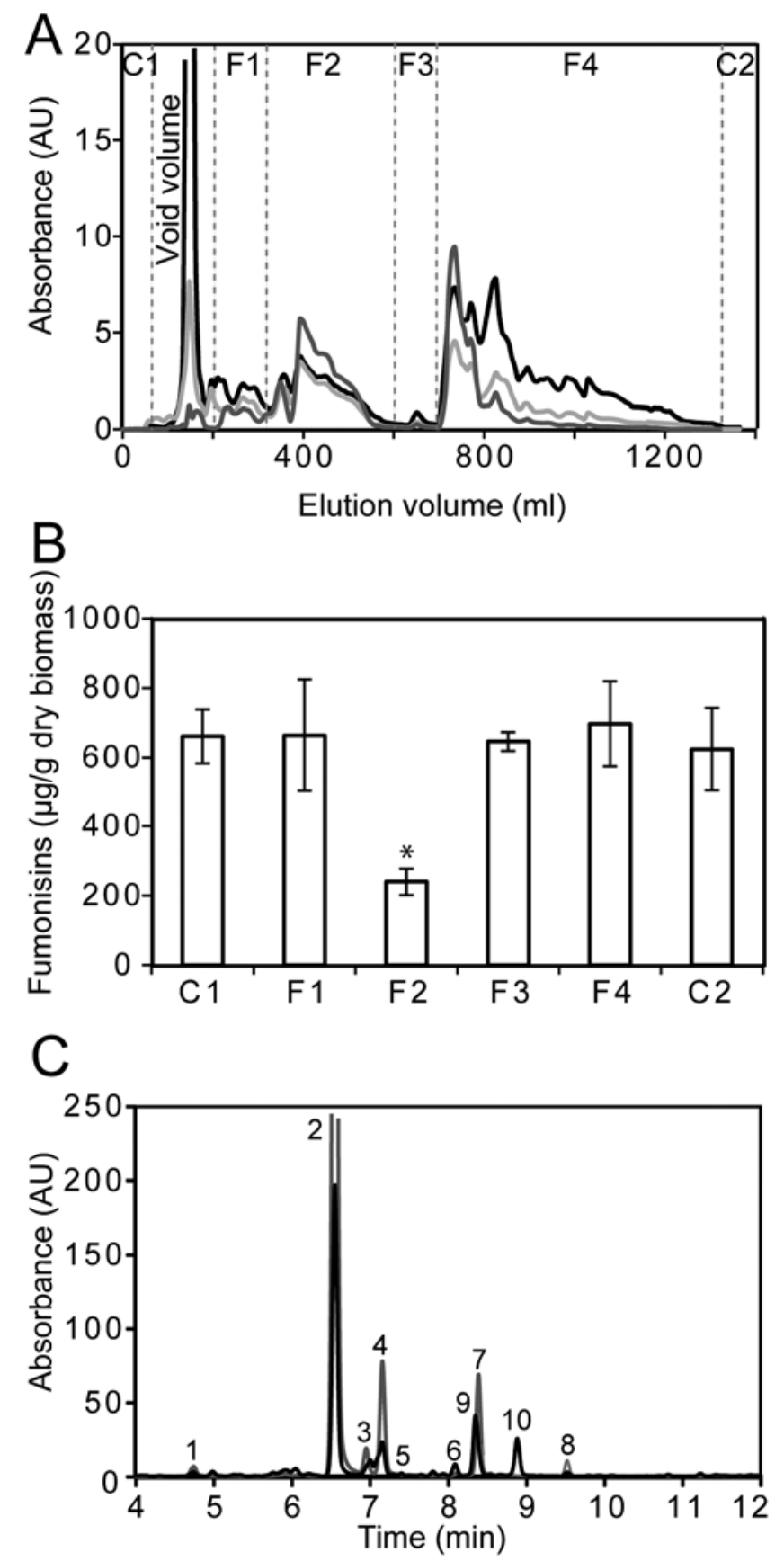

Fig. 1. Purification and characterization of inhibitory fraction for fumonisin accumulation by Fusarium verticillioides in maize kernels at milkdough stage. A, Elution profiles of methanol/water extract from a Fractogel gel filtration column at $230 \mathrm{~nm}$ (black), $280 \mathrm{~nm}$ (light gray), and 320 nm (dark gray). B, Effect of F1, F2, F3, and F4 fractions on fumonisin accumulation by $F$. verticillioides $\mathrm{Fv} 63 . \mathrm{C} 1$ and $\mathrm{C} 2$ are negative controls of $\mathrm{F} 1$ and $\mathrm{F} 2$ and $\mathrm{F} 3$ and $\mathrm{F} 4$ fractions, respectively; * indicates significant difference with control treatment $(\alpha=0.05)$. Data are means \pm standard deviation on three biological replicates. C, Chromatographic profiles at $320 \mathrm{~nm}$ (gray) and $260 \mathrm{~nm}$ (black) of F2 fraction. Peak $1=3-O$-caffeoylquinic acid, $2=$ trans $-5-O$-caffeoylquinic acid, $3=4-O$-caffeoylquinic acid, 4 = caffeic acid, $5=$ cis-5- $O$-caffeoylquinic acid or $1-O$-caffeoylquinic acid, $6=3$ - $O$-feruloylquinic acid, $7=5$-para-coumaroylquinic acid, $8=5$ $O$-feruloylquinic acid, $9=2-\beta$-D-glucopyranosyloxy-7-methoxy-1,4benzoxazin-3-one (HMBOA-Glc), and $10=2-\beta$-D-glucopyranosyloxy-4hydroxy-7-methoxy-1,4-benzoxazin-3-one (DIMBOA-Glc). 
$320 \mathrm{~nm}$ are reported in Figure 1C. Eight peaks (1, 2, 3, 4, 5, 6, 7 , and 8) absorbing at both 260 and $320 \mathrm{~nm}$ were visualized, as well as two additional ones (9 and 10) that only absorbed at $260 \mathrm{~nm}$. The 10 peaks were characterized by LC electrospray ionization mass spectrometry (ESI/MS) in negative or positive ionization modes. Their chromatographic and MS characteristics are summarized in Table 1 . Two of the major peaks detected at $320 \mathrm{~nm}$, peaks 2 and 4, exhibited UV-visible spectra typical of cinnamic acid derivatives (i.e., a maximum wavelength at $324 \mathrm{~nm}$ and a shoulder at $290 \mathrm{~nm}$ ). LC-MS analysis in negative-ion mode gave signals at $\mathrm{m} / \mathrm{z} 353$ and 179 for peaks 2 and 4, respectively. $\mathrm{MS}^{2}$ fragmentation of $\mathrm{m} / \mathrm{z} 353$ yielded product ions at $m / z, 191,179$, and 135, with the quinic acid moiety $(\mathrm{m} / \mathrm{z}, 191)$ representing the base peak. Based on these characteristics, peak 2 was assigned to 5-O-caffeoylquinic acid (5-CQA [chlorogenic acid]) and peak 4 was identified as caffeic acid (CA). These identifications were unequivocally confirmed by comparison with retention times and mass spectra of reference standards. Three others peaks (1,3, and 5) presented deprotonated molecule $[\mathrm{M}-\mathrm{H}]^{-}$at $\mathrm{m} / \mathrm{z} 353$ (Table 1). Fragmentation patterns of peaks 1 and 3 were in accordance with those assigned to 3-O-caffeoylquinic acid (3-CQA) and 4- $O$-caffeoylquinic acid (4-CQA) in literature data (Clifford et al. 2003). Although fragmentation of 3-CQA yielded a base peak resulting from quinic acid moiety $(\mathrm{m} / \mathrm{z} 191)$ and two intense signals from $\mathrm{CA}(\mathrm{m} / \mathrm{z}, 179)$ and $\left[\mathrm{CA}-\mathrm{H}-\mathrm{CO}_{2}\right]^{-}(\mathrm{m} / \mathrm{z}, 135)$, 4-CQA produced [quinic acid- $\left.\mathrm{H}_{-}-\mathrm{H}_{2} \mathrm{O}\right]^{-}$at $\mathrm{m} / z .173$ as base peak and two signals resulting from $\mathrm{CA}$ at $\mathrm{m} / \mathrm{z} 179$ and [CA$\left.\mathrm{H}-\mathrm{CO}_{2}\right]^{-}(\mathrm{m} / \mathrm{z}, 135)$ in the $\mathrm{MS}^{2}$ experiment. The third compound displaying an $\mathrm{m} / \mathrm{z}$ signal at 353 (peak 5) showed a fragmentation pattern identical to that of 5-CQA in $\mathrm{MS}^{2}$ negative-ion mode. We supposed that this compound is a cisisomer of 5-CQA or 1-CQA, because both are reported to fragment identically to trans-5-CQA. Phenolic cinnamic acids occur naturally in the trans configuration but exposure to UV light can induce photochemical isomerization to the cis con- figuration (Clifford et al. 2008). Peak 8 has a UV-visible spectrum typical of ferulic acid derivatives. LC/DAD-MS analysis in negative-ion mode gave a signal at $\mathrm{m} / \mathrm{z} 367$, and the mass chromatogram extracted at $\mathrm{m} / \mathrm{z}, 367$ from the total ion current revealed another, less important peak eluted at 8.1 min (peak $6)$. On the basis of UV-visible spectra and tandem mass spectrometry (MS/MS) fragmentation patterns, these compounds (peaks 6 and 8) were identified as two feruloylquinic acid isomers (Alonso-Salces et al. 2009; Clifford et al. 2003). Each isomer yielded a characteristic base peak ion: the first at $m / z$ 193 due to [ferulic acid $-\mathrm{H}]^{-}$ion and the second at $\mathrm{m} / \mathrm{z} 191$ due to [quinic acid-H] $]^{-}$ion. Thus, peak 6 was assigned as 3-Oferuloylquinic acid (3-FQA) and peak 8 as 5 - $O$-feruloylquinic acid (5-FQA), in accordance with a previous study (Figs. 1C and 2; Table 1) (Alonso-Salces et al. 2009). The compound eluting in peak 7 presented a deprotonated molecular ion at $\mathrm{m} / \mathrm{z} 337$ and its $\mathrm{MS}^{2}$ fragmentation yielded ions at $\mathrm{m} / \mathrm{z} 191$ compatible with [quinic acid-H] ${ }^{-}$and at $m / z, 93$ consistent with [phenoxide] $]^{-}$This compound was tentatively identified as 5- $p$ coumaroylquinic acid (5- $p$-CoQA). According to literature (Clifford et al. 2003), $p$-CoQA isomers behave similarly to feruloylquinic acid isomers, yielding the same kind of ions as $\mathrm{MS}^{2}$ base peak. Interpretation of this mass signal was supported by the UV-visible spectrum corresponding to $p$-coumaroyl derivatives with a maximum wavelength absorbance at $310 \mathrm{~nm}$ and a shoulder at $286 \mathrm{~nm}$ (Figs. 1C and 2; Table 1).

In addition to cinnamoyl and cinnamoylquinic acids, the chromatogram recorded at $260 \mathrm{~nm}$ revealed the presence of two peaks (Fig. 1C, peaks 9 and 10), showing UV-visible spectra characteristic of benzoxazinone derivatives (Dixon et al. 2012). Compound eluted in peak 9 presented a deprotonated molecular ion at $\mathrm{m} / \mathrm{z}$ 356. The fragmentation spectrum of $\mathrm{m} / \mathrm{z}$ 356 showed a loss of $162 \mathrm{mu}(\mathrm{m} / \mathrm{z}, 194)$ corresponding to a molecule loss of hexose. Thereafter, two successive losses of $28 \mathrm{mu}$ corresponding to $\mathrm{CO}$ were observed $(\mathrm{m} / \mathrm{z}, 166$ and $\mathrm{m} / \mathrm{z}$ 138), followed by a loss of $15 \mathrm{mu}$ corresponding to a methyl

Table 1. Chromatographic and mass spectrometry (MS) data for the identified compounds

\begin{tabular}{|c|c|c|c|c|c|c|}
\hline Ion mode & Number & $\begin{array}{l}\text { Retention time } \\
\text { (min) }\end{array}$ & Assignment $^{\mathrm{a}}$ & $\begin{array}{c}\text { UV-visible } \\
\text { spectrum }(\mathbf{n m})^{b}\end{array}$ & $\begin{array}{l}\text { Precursor } \\
\text { ion } m / z\end{array}$ & $\begin{array}{l}\text { MS }^{2} \text { cone voltage: } \pm 30 \mathrm{~V} \\
\text { collision energy: } \pm 30 \mathrm{eV} \mathrm{m} / \mathrm{z}(\%)\end{array}$ \\
\hline \multicolumn{7}{|l|}{$\mathrm{F} 2$ fraction $^{\mathrm{c}}$} \\
\hline Negative & 1 & 4.7 & 3-CQA & 290sh, 324 & 353.1 & $191(100), 179(48), 135(46)$ \\
\hline Negative & 2 & 6.5 & trans-5-CQA & 290sh, 324 & 353.1 & $191(100), 179(2), 135(1)$ \\
\hline Positive & 2 & 6.5 & trans-5-CQA & 290sh, 324 & 355.0 & $163(100), 145(12), 135(3)$ \\
\hline Negative & 3 & 6.9 & 4-CQA & 290sh, 324 & 353.1 & $179(40), 173(100), 135(44)$ \\
\hline Negative & 4 & 7.1 & $\mathrm{CA}$ & 290sh, 324 & 179.1 & $135(100), 117(2)$ \\
\hline Negative & 5 & 7.4 & cis-5-CQA or 1-CQA & $*$ & 353.1 & 191(100), 179(2) \\
\hline Negative & 6 & 8.1 & 3-FQA & $*$ & 367.0 & $193(100), 134(53)$ \\
\hline Negative & 7 & 8.4 & 5- $p$-CoQA & 286 sh, 310 & 337.2 & $191(100), 93(11)$ \\
\hline Negative & 8 & 9.6 & 5-FQA & $286 \mathrm{sh}, 325$ & 367.0 & 191(100), 173(34), 134(8), 93(13) \\
\hline Negative & 9 & 8.3 & HMBOA-Glc & $265,285 \mathrm{sh}^{\mathrm{d}}$ & 356.0 & $194(100), 166(64), 138(75), 123(28)$ \\
\hline Negative & 10 & 8.9 & DIMBOA-Glc & $250,285 \mathrm{sh}$ & 372.1 & $164(17), 149(100)$ \\
\hline \multicolumn{7}{|l|}{ Fv $63^{e}$} \\
\hline Negative & $\ldots$ & 3.4 & $\mathrm{PA}^{\mathrm{f}}$ & 260,292 & 153.0 & \\
\hline Negative & $\ldots$ & 3.6 & trihydroxyCINQA ${ }^{\mathrm{g}}$ & 324 & 369.0 & $191(100)$ \\
\hline Positive & $\ldots$ & 3.6 & trihydroxyCINQA ${ }^{g}$ & 324 & 371.0 & 179(85), 161(30), 133(100) \\
\hline Negative & $\ldots$ & 3.9 & hydroxyCA ${ }^{f}$ & 324 & 195.0 & $151(100), 97(74), 79(98)$ \\
\hline Negative & $\ldots$ & 6.5 & trans-5-CQA ${ }^{\mathrm{g}}$ & 290sh, 324 & 353.1 & 191(100), 179(2), 135(1) \\
\hline Positive & $\ldots$ & 6.5 & trans-5-CQA ${ }^{\mathrm{g}}$ & $290 \mathrm{sh}, 324$ & 355.0 & $163(100), 145(12), 135(3)$ \\
\hline Negative & $\ldots$ & 7.1 & $\mathrm{CA}^{\mathrm{f}, \mathrm{g}}$ & $290 \mathrm{sh}, 324$ & 179.1 & $135(100), 117(2)$ \\
\hline
\end{tabular}

a 5-CQA = 5- $O$-caffeoylquinic acid, 3-CQA = 3- $O$-caffeoylquinic acid, $1-\mathrm{CQA}=1-O$-caffeoylquinic acid, $\mathrm{CA}=\mathrm{caffeic}$ acid, $\mathrm{PA}=$ protocatechuic acid, $\mathrm{FQA}=$ feruloylquinic acid, $p$-CoQA $=p$-coumaroylquinic acid, trihydroxyCINQA $=$ trihydroxycinnamoylquinic acid, hydroxyCA $=$ hydroxycaffeic acid, $\mathrm{BOA}=$ benzoxazolin-2-one, HMBOA-Glc = 2- $\beta$-D-glucopyranosyloxy-7-methoxy-1,4-benzoxazin-3-one, and DIMBOA-Glc = 2- $\beta$-D-glucopyranosyloxy-4hydroxy-7-methoxy-1,4-benzoxazin-3-one.

${ }^{\mathrm{b}}$ Asterisks $(*)$ indicate compounds hidden by others or in traces. Their UV-visible spectra have not been properly observed.

${ }^{\mathrm{c}}$ Compounds detected in active F2 fraction purified from maize kernels.

${ }^{\mathrm{d}}$ UV-visible spectra of $7+9$.

e Compounds detected in liquid cultures of Fusarium verticillioides Fv 63 (supernatants and mycelia).

${ }^{\mathrm{f}}$ Compound detected in liquid cultures of $F$. verticillioides Fv 63 (supernatants and mycelia) supplemented with caffeic acid at 0.5 mM.

g Compound detected in liquid cultures of F. verticillioides Fv 63 (supernatants and mycelia) supplemented with chlorogenic acid at 0.5 mM. 
group $(\mathrm{m} / \mathrm{z}$ 123). Based on these characteristics and reported literature data (Bonnington et al. 2003; Cambier et al. 1999), this compound was identified as 2- $\beta$-D-glucopyranosyloxy-7methoxy-1,4-benzoxazin-3-one (HMBOA-Glc) (Fig. 2; Table 1). LC/DAD-MS analysis in negative-ion mode of peak 10 allowed a signal at $\mathrm{m} / \mathrm{z}, 372$. This observation was confirmed by the presence of a formic adduct at $\mathrm{m} / \mathrm{z} 418$. Fragmentation of $\mathrm{m} / \mathrm{z}, 372$ ion yielded two fragment ions at $\mathrm{m} / \mathrm{z}, 164$ and 149. The ion at $m / z, 164$ can be assigned to the fragment arising from the loss of the glycosylated carbon and hydroxyl group at the nitrogen. The ion at $\mathrm{m} / z, 149$ corresponds to a subsequent loss of $15 \mathrm{mu}$ from the $\mathrm{m} / \mathrm{z}, 164$, assignable to the cleavage of a methyl group. On the basis of these results and literature data (Bonnington et al. 2003), this molecule was putatively assigned to 2- $\beta$-D-glucopyranosyloxy-4-hydroxy-7-methoxy1,4-benzoxazin-3-one (DIMBOA-Glc) (Fig. 2; Table 1).

To summarize, 10 compounds were unequivocally (5-CQA and $\mathrm{CA}$ ) or putatively identified in the F2 fraction. Eight compounds are cinnamoyl or cinnamoylquinic acid derivatives and two compounds belong to the benzoxazinone family, the HMBOA-Glc and the DIMBOA-Glc. 5-CQA, the concentration of which was close to $404 \mathrm{mg} / \mathrm{g}$ of lyophilized F2 fraction, was clearly the most abundant molecule. Ranked by decreasing concentrations, the other quantified compounds were HMBOAGlc (23 mg/g), DIMBOA-Glc (18.2 mg/g), CA, and 5- $p$-CoQA $(12.3 \mathrm{mg} / \mathrm{g}$ each) and, last, 5-FQA $(6 \mathrm{mg} / \mathrm{g})$.

In vitro effect of 5-CQA on fumonisin biosynthesis.

The effect of $0.5 \mathrm{mM} 5-\mathrm{CQA}$ on fungal development and fumonisin biosynthesis was investigated in liquid cultures using six strains of $F$. verticillioides and four strains of $F$. proliferatum (Table 2). This concentration is consistent with physiological levels previously determined in maize kernels at its earliest stages of development (Atanasova-Penichon et al. 2012). Fusarium strains were chosen according to amount of fumonisin they can produce: low level (Fv 62, Fp 393, and Fp 381), average level (Fp 58, Fv 444, and Fp 367) and high level (Fv 63, Fv 64, Fv 368, and Fv 424).

FB1 was the major fumonisin produced by the studied strains, in accordance with previous reports (Sanchez-Rangel et al. 2005). The FB2/FB1 and FB3/FB1 ratios were not significantly affected by the treatment (data not shown). Therefore, the sum $\mathrm{FB} 1+\mathrm{FB} 2+\mathrm{FB} 3$ can be used to quantify the amount of fumonisins produced. 5-CQA treatment did not affect fungal biomass production by any of the isolates, in our conditions (Table 2). On the other hand, 5-CQA induced a significant reduction in fumonisin accumulation for seven of the studied isolates. High inhibition efficiency was observed for four strains ( $\mathrm{Fp} \mathrm{58,}$ Fp 393, Fp 367, and Fv 62), with more than $90 \%$ of fumonisin reduction after 15 days of culture. For three isolates $(\mathrm{Fv} 63, \mathrm{Fv}$ 64, and Fv 444), the decrease in fumonisin accumulation was less drastic (close to 50\%). Therefore, 5-CQA is an effective inhibitor of fumonisin biosynthesis by $F$. verticillioides and $F$. proliferatum, with an inhibition efficiency that varies according to the considered strain. We further investigated the mechanisms involved in fumonisin inhibition by 5-CQA and examined the metabolic fate of this phenolic acid when supplemented to $F$. verticillioides broths. Given the reported ability of filamentous fungi to hydrolyze 5-CQA, yielding CA and quinic acid (TorresMancera et al. 2013), CA was also considered.

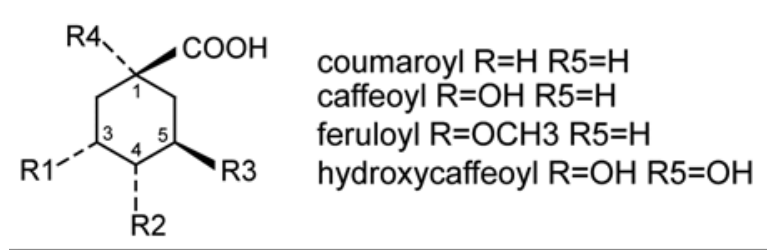

\begin{tabular}{|l|c|c|c|c|}
\hline Compound name & R1 & R2 & R3 & R4 \\
\hline Quinic acid & OH & OH & OH & OH \\
\hline 1-O-caffeoylquinic acid & OH & OH & OH & caffeoyl \\
\hline 3-O-caffeoylquinic acid & caffeoyl & OH & OH & OH \\
\hline 4-O-caffeoylquinic acid & OH & caffeoyl & OH & OH \\
\hline 5-O-caffeoylquinic acid & OH & OH & caffeoyl & OH \\
\hline Hydroxychlorogenic acid & OH & OH & hydroxycaffeoyl & OH \\
\hline 3-O-feruloylquinic acid & feruloyl & OH & OH & OH \\
\hline 5-O-feruloylquinic acid & OH & OH & feruloyl & OH \\
\hline 5-p-coum aroylquinic acid & OH & OH & coum aroyl & OH \\
\hline
\end{tabular}

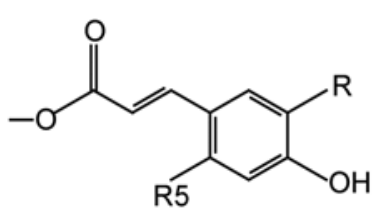<smiles>[R1]N1C(=O)C(O[Ge])Oc2cc(OC)ccc21</smiles>

HMBOA-Glc R1=H DIMBOA-GIC R1=OH

Fig. 2. Structure of the compounds identified in this study. HMBOA-Glc $=2-\beta$-D-glucopyranosyloxy-7-methoxy-1,4-benzoxazin-3-one and DIMBOA-Glc $=$ 2- $\beta$-D-glucopyranosyloxy-4-hydroxy-7-methoxy-1,4-benzoxazin-3-one.

Table 2. Effect of $0.5 \mathrm{mM}$ chlorogenic acid (5-CQA) on fungal biomass and fumonisin accumulation by 10 isolates of Fusarium $^{\mathrm{a}}$

\begin{tabular}{lcccc}
\hline & \multicolumn{2}{c}{ FB1 + FB2 + FB3 $(\boldsymbol{\mu g} / \mathbf{g}$ of dry biomass) } & \multicolumn{2}{c}{ Dry biomass (mg) } \\
\cline { 2 - 5 } Strain & Control & 5-CQA & Control & 5-CQA \\
\hline Fv 62 & $244.6 \pm 34.4$ & $19.2 \pm 6.5^{*}$ & $90.6 \pm 17.2$ & $84.4 \pm 19.0$ \\
Fv 63 & $1,965.8 \pm 58.4$ & $1,060.4 \pm 83.8^{*}$ & $72.8 \pm 12.0$ & $79.4 \pm 3.8$ \\
Fv 64 & $2,590.7 \pm 332.8$ & $1,433.7 \pm 108.2^{*}$ & $81.9 \pm 25.0$ & $98.14 \pm 5.0$ \\
Fv 368 & $2,394.2 \pm 241.8$ & $2,424.7 \pm 399.3$ & $108.7 \pm 6.0$ & $110.1 \pm 14.7$ \\
Fv 424 & $3,479.9 \pm 471.4$ & $4,724.8 \pm 1,401.6$ & $81 \pm 8.4$ & $71.8 \pm 5.0$ \\
Fv 444 & $691.9 \pm 188.8$ & $284.2 \pm 34.3^{*}$ & $99.3 \pm 11.0$ & $88.5 \pm 9.7$ \\
Fp 58 & $533.6 \pm 134.3$ & $5.6 \pm 2.1^{*}$ & $75.1 \pm 15.2$ & $93.8 \pm 8.1$ \\
Fp 367 & $598.6 \pm 118.8$ & $29.1 \pm 6.5^{*}$ & $101 \pm 20.9$ & $95.5 \pm 14.1$ \\
Fp 381 & $142.6 \pm 38.5$ & $100.3 \pm 58.4$ & $76.9 \pm 5.9$ & $68.7 \pm 7.2$ \\
Fp 393 & $62.4 \pm 35.1$ & $2.3 \pm 1.2^{*}$ & $103 \pm 12.0$ & $82 \pm 12.2$ \\
\hline
\end{tabular}

a Asterisk (*) indicates significant difference with control treatment $(\alpha=0.05)$. Data are means \pm standard deviation using three biological replicates. 


\section{Biotransformation of 5-CQA and CA when supplemented} to liquid cultures of $F$. verticillioides $F v 63$.

5-CQA or CA at $0.5 \mathrm{mM}$ were supplemented in Fv 63 liquid cultures. Fungal growth, fumonisin accumulation, and phenolic acid metabolic fate were monitored over time, up to 21 days after inoculation. Fungal growth was not affected by the phenolic acid treatment with 5-CQA or CA (Fig. 3). Biomass is detected as soon as $24 \mathrm{~h}$ after inoculation and increases sharply until the third day of culture (exponential phase of growth). Then, kinetics of biomass accumulation slow down and reach the stationary phase of growth after 4 days of culture. Regarding fumonisin accumulation in supernatants (Fig. 3 ), toxins were first quantified $48 \mathrm{~h}$ after inoculation. Fumonisin production was nearly linear between days 4 and 21 , toxin yields reaching approximately $5,000 \mu \mathrm{g} / \mathrm{g}$ in nonsupplemented cultures. After 21 days of incubation, fumonisin

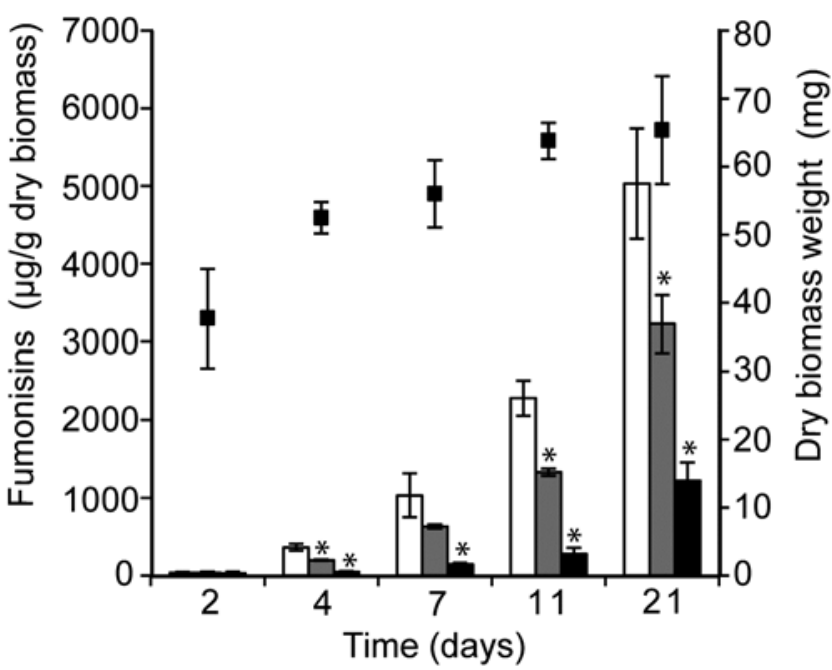

Fig. 3. Kinetics of fumonisin and biomass accumulation in liquid cultures of Fusarium verticillioides Fv 63 supplemented or not with $0.5 \mathrm{mM}$ chlorogenic or caffeic acid. Bars (left $y$ axis) show fumonisin levels in cultures supplemented with chlorogenic acid (gray) or caffeic acid (black) or nonsupplemented (white). Squares show biomass accumulation in not supplemented cultures (right $y$ axis); * indicates significant differences when compared with control treatment $(\alpha=0.05)$. Data are means \pm standard deviation using three biological replicates. levels were reduced by 36 and $76 \%$ in the presence of 5-CQA and CA, respectively. When assayed in mycelia, fumonisins were first detected in 2-day-old mycelia and their concentration increased regularly over time to attain a value close to 80 $\mu \mathrm{g} / \mathrm{g}$ in 21-day-old mycelia. This level did not significantly vary according to the treatment (data not shown). These results support the significant inhibitory activity of 5-CQA and highlight the high efficiency of CA in limiting toxin accumulation.

5-CQA and CA metabolic rates were followed, using LC/DAD and LC/ESI-MS, in supplemented cultures of $F$. verticillioides $\mathrm{Fv} 63$ as well as noninoculated supplemented broths. In noninoculated media, levels of 5-CQA and CA remained stable throughout the 21 days of culture (data not shown). In Fv 63-inoculated and 5-CQA-supplemented cultures, a slight decrease in 5-CQA, less than $10 \%$ of the initial quantity, was observed in the supernatants in up to 7-day-old cultures. This decline increased after 7 days, and less than $25 \%$ of the initial 5-CQA was detected after 21 days of culture in the supernatants (Table 3). The small amount of 5-CQA that had disappeared from the supernatants during the first days of culture was found in the mycelia. However, after 7 days of culture, only traces of 5-CQA were quantified in mycelia, which could not explain the 5-CQA decrease in the supernatants (Table 3). This unbalanced ratio suggests the occurrence of a metabolic transformation of 5-CQA. In addition to 5-CQA, two major peaks were detected in the LC/DAD profiles of both culture supernatants and mycelia at $320 \mathrm{~nm}$. One of them was unequivocally identified as $\mathrm{CA}$, on the basis of its retention time and UV-visible spectrum compared with a commercial standard (Table 1). The second peak, characterized by a UVvisible spectrum typical of cinnamic acid derivatives $\left(\lambda_{\max }\right.$ at $324 \mathrm{~nm}$ ), is associated with an $\mathrm{m} / \mathrm{z} 369$ ion when analyzed by LC/ESI-MS in negative-ion mode. This $\mathrm{m} / z, 369$ signal is consistent with the addition of one hydroxyl group $(16 \mathrm{mu})$ to 5 CQA. Its MS/MS fragmentation in negative-ion mode gave one signal at $m / z$ 191, probably due to the deprotonated quinic moiety (Supplementary Fig. 1). In positive-ion mode, the MS/MS fragments were at $\mathrm{m} / z, 179[\mathrm{M}+\mathrm{H}-192]^{+}, 161[\mathrm{M}+\mathrm{H}-$ $192-18]^{+}$, and $133[\mathrm{M}+\mathrm{H}-192-28-18]^{+}$. This fragmentation behavior is similar to that observed for 5-CQA in positive-ion mode. Thus, on the basis of UV-visible spectrum and MS/MS fragmentation patterns, we putatively identified this compound as trihydroxycinnamoylquinic acid (trihydroxyCINQA) or

Table 3. Kinetics of chlorogenic acid, caffeic acid, and their metabolites in the supernatants and mycelia of cultures of Fusarium verticillioides Fv 63 supplemented with $0.5 \mathrm{mM}$ chlorogenic or caffeic acid ${ }^{\mathrm{a}}$

\begin{tabular}{|c|c|c|c|c|c|c|}
\hline \multirow[b]{2}{*}{ Culture (days) ${ }^{b}$} & \multicolumn{3}{|c|}{ Chlorogenic acid-supplemented cultures } & \multicolumn{3}{|c|}{ Caffeic acid-supplemented cultures } \\
\hline & Compound ( $\mu \mathrm{mol})$ & Supernatant & Mycelium & Compound ( $\mu \mathrm{mol})$ & Supernatant & Mycelium \\
\hline 0 & Chlorogenic acid & 5 & 0 & Caffeic acid & 5 & 0 \\
\hline 2 & & $4.6930 \pm 0.1551$ & $0.1860 \pm 0.0388$ & & $0.3851 \pm 0.0780$ & $0.0065 \pm 0.0024$ \\
\hline 4 & & $4.8135 \pm 0.2887$ & $0.0976 \pm 0.0411$ & & n.d. & n.d. \\
\hline 7 & & $4.5423 \pm 0.3783$ & $0.0452 \pm 0.0115$ & & n.d. & n.d. \\
\hline 11 & & $3.6327 \pm 0.4413$ & $0.0358 \pm 0.0086$ & & n.d. & n.d. \\
\hline 21 & & $1.2146 \pm 0.1776$ & $0.0168 \pm 0.0051$ & & n.d. & n.d. \\
\hline 0 & Caffeic acid & 0 & 0 & Protocatechuic acid & 0 & 0 \\
\hline 2 & & $0.0555 \pm 0.0111$ & $0.0044 \pm 0.0019$ & & $0.4285 \pm 0.1575$ & $0.0173 \pm 0.0069$ \\
\hline 4 & & $0.0555 \pm 0.0123$ & $0.0038 \pm 0.0016$ & & $0.0865 \pm 0.0074$ & n.d. \\
\hline 7 & & $0.0688 \pm 0.0093$ & $0.0029 \pm 0.0007$ & & n.d. & n.d. \\
\hline 11 & & $0.0791 \pm 0.0159$ & $0.0020 \pm 0.0001$ & & n.d. & n.d. \\
\hline 21 & & $0.0555 \pm 0.0192$ & $0.0027 \pm 0.0008$ & & n.d. & n.d. \\
\hline 0 & Hydroxychlorogenic acid & 0 & 0 & Hydroxycaffeic acid & 0 & 0 \\
\hline 2 & & $0.1153 \pm 0.0484$ & $0.0053 \pm 0.0026$ & & $0.1292 \pm 0.0212$ & $0.0072 \pm 0.0018$ \\
\hline 4 & & $0.1225 \pm 0.0573$ & $0.0026 \pm 0.0011$ & & $0.0884 \pm 0.0059$ & $0.0033 \pm 0.0004$ \\
\hline 7 & & $0.0689 \pm 0.0052$ & $0.0007 \pm 0.0001$ & & $0.0340 \pm 0.0294$ & $0.0018 \pm 0.0007$ \\
\hline 11 & & $0.1081 \pm 0.0507$ & $0.0004 \pm 0.0001$ & & $0.0136 \pm 0.0235$ & $0.0006 \pm 0.0001$ \\
\hline 21 & & $0.0900 \pm 0.0125$ & $0.0009 \pm 0.0004$ & & n.d. & $0.0004 \pm 0.0001$ \\
\hline
\end{tabular}

${ }^{\text {a }}$ Data are means \pm standard deviation on three biological replicates; n.d. $=$ not detected.

${ }^{\mathrm{b}}$ Culture duration. 
hydroxychlorogenic acid (Table 1; Fig. 2). When quantified in mycelia and culture supernatants, $\mathrm{CA}$ and hydroxychlorogenic acid were present at very low levels, close to 0.1 and 0.005 $\mu \mathrm{mol}$, respectively. These levels remained stable in culture supernatants between 2 and 21 days of culture (Table 3 ). These two compounds were not detected in noninoculated cultures supplemented with 5-CQA, indicating that the transformation of 5-CQA was caused by $F$. verticillioides.

Regarding CA, the LC-DAD data revealed a drastic decrease in the quantities found both in supernatants and mycelia during the first 4 days of cultures (Table 3 ). The LC-DAD profiles at 260 and $320 \mathrm{~nm}$ further revealed the occurrence of two other peaks. One of these two peaks was unequivocally identified as protocatechuic acid (PA) by comparison with retention time as well as UV-visible and mass spectra of a reference standard (Table 1; Fig. 2). The second peak was characterized by a UVvisible spectrum similar to that previously ascribed to hydroxychlorogenic acid. Mass spectrometry data obtained by LC/ESIMS in negative-ion mode showed a signal at $m / z$ 195. This $m / z$ 195 signal could correspond to the addition of one hydroxyl group (16 mu) to CA. Its MS/MS fragmentation in negativeion mode gave $\left[\mathrm{M}-\mathrm{H}-\mathrm{CO}_{2}\right]^{-}$at $\mathrm{m} / \mathrm{z}, 151$ as a base peak and two intense signals at $\mathrm{m} / \mathrm{z} 97$ and 79. On the basis of the UV-visible spectrum identical to that of hydroxychlorogenic acid and the MS/MS fragmentation pattern similar to that of CA, this compound was putatively identified as a hydroxycaffeic acid (hydroxyCA) (Table 1; Fig. 2). The maximum amounts of PA and hydroxyCA were quantified during the first 2 days of culture in supernatants and mycelia (Table 3). PA was detected in 2-day-old cultures, at $0.4 \mu \mathrm{mol}$ in supernatants, and rapidly disappeared between 4 and 7 days. HydroxyCA was detected in 2-day-old cultures, at $0.13 \mu \mathrm{mol}$ in supernatants, and a gradual decrease occurred from day 2 to 21 . These amounts, largely higher in supernatants than in mycelia, never exceeded $0.4 \mu \mathrm{mol}$ and could not explain the disappearance of $4.5 \mu \mathrm{mol}$ of CA observed after 2 days of incubation. Similarly to 5CQA-supplemented cultures, PA and hydroxyCA were not detected in noninoculated cultures supplemented with CA, indicating that this mechanism of transformation was caused by $F$. verticillioides.

\section{Relationship between Fusarium ear rot resistance level and 5-CQA content in immature maize kernels.}

A set of 10 maize genotypes divided into three groups according to their level of susceptibility to Fusarium ear rot (highly susceptible, susceptible, and moderately susceptible) was used to establish a relationship between resistance to Fusarium ear rot and 5-CQA amount in immature kernels. The maize inbred line used for the bioguided fractionation approach performed in the first part of this work is included in the moderately susceptible group. 5-CQA levels were found to greatly vary among genotypes and ranged between 5 and $908 \mu \mathrm{g} / \mathrm{g}$. As clearly appeared on the box plot representation reported in Figure 4, our data showed significant differences between the moderately susceptible group and the two other groups of genotypes: levels of 5-CQA were significantly higher in the moderately susceptible group.

\section{DISCUSSION}

The hypothesis that resistance to fumonisin accumulation in some maize genotypes can be explained by a particular biochemical composition of kernels, rich in specific endogenous metabolites affecting fumonisin biosynthesis, has been the subject of recent investigations (Campos-Bermudez et al. 2013; Picot et al. 2013). These works, coupled with earlier in vitro experiments (Beekrum et al. 2003; Dambolena et al.
2008; Samapundo et al. 2007), led to establishing a list of potential "active principles" that includes phenolic compounds, tocopherols, and carotenoids, as well as polyamines. To gain insights into the respective roles played by these potential "active principles", we developed a bioguided fractionation approach. We used kernels of a maize inbred with a moderate susceptibility to $F$. verticillioides, harvested at the milk-dough stage, which corresponds to the time fumonisin production starts in developing maize kernels (Picot et al. 2011).

\section{5-CQA in immature maize kernels is the "anti-fumonisin" compound.}

Among the extracts we obtained using solvents of different polarity strengths, the methanol/ $\mathrm{H}_{2} \mathrm{O}$ extract, which contained semipolar compounds, was the only one that induced a significant inhibition of fumonisin yields in vitro. Further fractionation and testing coupled to LC/DAD and LC/MS analyses revealed that 5-CQA was predominantly present in the only fraction with fumonisin inhibitory properties. Small amounts of seven others cinnamoyl or cinnamoylquinic acid derivatives and two compounds belonging to the benzoxazinone family, HMBOA-Glc and DIMBOA-Glc, were also detected in this fraction. Natural phenolic extracts and pure phenolic acids have frequently been reported as natural compounds that can inhibit mycotoxin production. For instance, natural phenolic acid extracts from maize germ (Bakan et al. 2003) or durum wheat bran (Boutigny et al. 2010) were shown to significantly repress in vitro type B trichothecene production. Phenolic extracts from the pellicle of walnut (Mahoney and Molyneux 2004) or from olive callus tissues (Paster et al. 1988) were also investigated for their efficiency in affecting aflatoxin production by Aspergillus spp. Regarding pure phenolic acids, the impact of CA and ferulic acid on mycotoxin production, including type B trichothecenes (Boutigny et al. 2009; Ponts et al. 2011) and fumonisins (Beekrum et al. 2003; Picot et al. 2013), has been the subject of several reports. In contrast, very few data describing the effect of 5-CQA are available. To assess whether the active fraction's inhibitory activity is caused by the presence of 5-CQA, we further investigated fumonisin production by $F$. verticillioides in 5-CQA-supplemented media. We demonstrated that 5-CQA significantly reduced fumonisin yields for 7 of the 10 strains tested, without affecting fungal biomass accumulation. At $0.5 \mathrm{mM}$, inhibition intensity was close to $45 \%$ for the model strain Fv 63 that we used throughout our study. When $500 \mathrm{mg}$ of the lyophilized active chromatographic fraction (containing $202 \mathrm{mg}$ of 5-CQA) was added to 1 liter of $\mathrm{Fv} 63$ culture medium, which corresponds to a

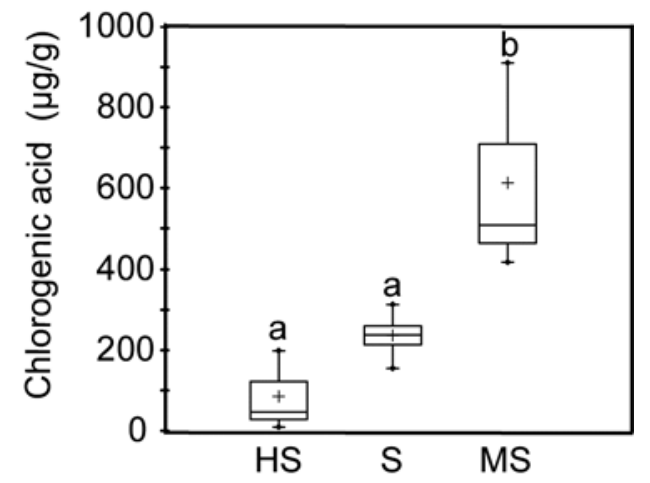

Fig. 4. Chlorogenic acid levels in immature kernels from three groups of genotypes differing in their susceptibility to Fusarium ear rot: highly susceptible (HS, $n=3$ ), susceptible (S, $n=4)$, and moderately susceptible (MS, $n=3$ ). Groups with different letters are significantly different (Duncan, $\alpha=0.05)$. 
final concentration of 5-CQA close to $0.5 \mathrm{mM}$, inhibition intensity reached $65 \%$. Therefore, our data indicate that 5CQA abundance in the active chromatographic fraction could be responsible for $70 \%$ of its inhibitory activity. This $70 \%$ theoretical value does not take into account the potential occurrence of synergistic or antagonistic interactions that could result from the co-occurrence of 5-CQA and additional compounds in the active chromatographic fraction and could significantly modulate the inhibition efficiency of 5-CQA. Synergistic inhibitory effects on ochratoxine A production by Aspergillus carbonarius and $A$. niger were recently highlighted by Barberis and associates (2010) using binary mixtures of antioxidants. The residual inhibitory activity in the active fraction could also be linked to the presence of the others compounds we identified in the chromatographic extract in addition to 5CQA but also to compounds that our analysis could not identify. Free cinnamoyl and cinnamoylquinic acids, present at low amounts, belong to the same family as CA and 5-CQA and are likely to be efficient fumonisin biosynthesis inhibitors. With regards to DIMBOA-Glc and HMBOA-Glc, the ability shown by some benzoxazinoid hydroxamic acids to repress trichothecene and aflatoxin production has been reported (Miller et al. 1996) but no data on their potential impact on fumonisin production are available. However, benzoxazinoid hydroxamic acids have been extensively studied for their antimicrobial efficiency and their key role in defense of maize against various pathogens (Ahmad et al. 2011; Niemeyer 2009). Although benzoxazinoid hydroxamic acids are able to inhibit the growth of various fungal pathogens, including $F$. graminearum and $F$. verticillioides along with other Fusarium spp. (Glenn et al. 2001), their role in fungal resistance remains ambiguous. On one hand, positive correlations were found between resistance rating of wheat cultivars to Puccinia graminis and benzoxazinoid hydroxamic acid concentration at the seedling stage (Zheng et al. 2005), as well as between Fusarium head blight susceptibility and benzoxazinoid hydroxamic acid concentration in wheat ears (Soltoft et al. 2008). On the other hand, severity of corn smut caused by Ustilago zeae was not correlated with benzoxazinoid hydroxamic acid concentration in seedlings (Reid et al. 1991). Similarly, the role of benzoxazinoid hydroxamic acids in maize resistance to $F$. verticillioides was shown to be unimportant, possibly due to the fungus's ability to metabolically transform benzoxazinoid hydroxamic acids into nontoxic metabolites (Glenn et al. 2002).

Nonetheless, results of the bioguided fractionation strategy used here highlight, for the first time, the key role that 5-CQA may play in fumonisin resistance. This key role was corroborated by the relationship between resistance to Fusarium ear rot and 5-CQA content in immature kernels that we showed using nine additional genotypes. The contribution of 5-CQA to resistance mechanisms has also been previously reported in different pathosystems, including chrysanthemum-thrips (Leiss et al. 2009), peach-Monilia laxa (Villarino et al. 2011), and, more recently, maize- $F$. graminearum (Atanasova-Penichon et al. 2012). Our results are new arguments in favor of the involvement of plant phenolic compounds in constitutive defense mechanisms (Lattanzio et al. 2006). Cell-wall-bound ferulic acid has also been reported to participate as a constitutive defense compound to Fusarium ear rot resistance (Assabgui et al. 1993; Picot et al. 2013). In our study, we decided to focus on free phenolic compounds that are more likely to be encountered by the invading fungal pathogens and have been reported as more toxic than the bound forms (Lattanzio et al. 2006). Therefore, the hydrolysis step required to extract bound phenolic acids, including ferulic acid, was not introduced in the extraction procedure we used, which explains that ferulic acid was not retrieved in the active chromatographic fraction.
Metabolic transformation of 5-CQA by $F$. verticillioides.

Little is known about metabolic pathways of phenolic acid biotransformations by Fusarium spp. Knowledge is mainly restricted to feruloyl esterases enzymes, also known as ferulic acid esterases. Feruloyl esterases are subclasses of carboxylic acid esterases and include 5-CQA hydrolase. These enzymes are well known for their ability to cleave the ester linkages between hydroxycinnamic acids and the plant cell wall. They can also catalyze the hydrolysis of 5-CQA into CA and quinic acid. The occurrence of feruloyl esterase activities, frequently described in several Aspergillus spp. (Asther et al. 2005; Benoit et al. 2007; Torres-Mancera et al. 2013), was reported in F. proliferatum and F. verticillioides (Shin and Chen 2006). The phylogenic tree established in 2012 by Balcerzak and associates (2012) revealed the occurrence of five genes coding for feruloyl esterase or related proteins in the genome of $F$. verticillioides. Elucidating the ability of $F$. verticillioides to biotransform 5-CQA could provide new information on the mechanism by which this phenolic acid affects fumonisin production but also new arguments in favor of its involvement in fumonisin resistance in planta. Here, our results highlight the occurrence of biotransformation pathways for 5-CQA in F. verticillioides, which was supported by the detection and quantification of 5-CQA metabolites CA and hydroxychlorogenic acid (Fig. 5). The presence of CA, which can result from 5-CQA hydrolysis catalyzed by a feruloyl esterase, is consistent with the occurrence of such enzymatic activities in F. verticillioides (Shin and Chen 2006). Hydroxychlorogenic acid can result from the enzymatic hydroxylation of 5-CQA, according to a pathway previously described for CA (Kader et al. 2001) or 4-methylcatechol (Richard-Forget et al. 1992). Given the relationship established between lipophilicity and fungal toxicity of phenolic compounds (Guiraud et al. 1995), hydrolysis of 5-CQA into CA could lead to a more toxic metabolite whereas hydroxylation of 5-CQA could result in a less toxic molecule. However, the amounts of 5-CQA metabolites we quantified in supernatants and mycelia were not equivalent to the loss of 5-CQA, which suggests additional, as-yet-undetected 5-CQA biotransformation pathways or further biotransformations of 5-CQA metabolites. Considering that the $\mathrm{pH}$ of our liquid cultures was higher than 4.5 , we cannot exclude the occurrence of 5-CQA oxidation reactions even though we did not detect 5-CQA oxidation products with the LC method used here. Up to nine putative laccase or related genes were identified in the genome of $F$. verticillioides according to a quick search in Fungal Genetic Database, which illustrates the potential ability of the fungus to oxidize phenolic compounds.

Our results have also demonstrated a rapid biotransformation of $\mathrm{CA}$ in $F$. verticillioides liquid cultures into $\mathrm{PA}$ and hydroxyCA. The present work is the first demonstration of the ability of $F$. verticillioides to biotransform and detoxify CA, a potent antifungal phenolic compound (Guiraud et al. 1995) (Fig. 5). Degradation of CA into PA could come from two different pathways that have been recently described in A. niger cultures (Torres-Mancera et al. 2013): i) CA transformation into caffeoyl-CoA by caffeoyl-CoA synthase and into PA by enoylCoA-hydratase and hydrogenase and ii) transformation into $p$ coumaric acid followed by decarboxylation and, finally, formation of PA. In our study, $p$-coumaric acid was never detected, suggesting that biotransformation of $\mathrm{CA}$ by $F$. verticillioides predominantly follows the first pathway. PA was further shown to be used as a carbon source by A. niger and transformed into different final products incorporated into the metabolic pathway (Torres-Mancera et al. 2013). Our study did not allow conclusions on the occurrence of this additional pathway in F. verticillioides cultures.

As a whole, our data indicate that in planta fumonisin production in maize could be reduced by the presence of naturally 
occurring 5-CQA. Indeed, our results demonstrate that 5-CQA is the major bioactive compound in kernels from moderately susceptible maize varieties, harvested at the milk-dough stage (i.e., at the starting kernel stage for fungal colonization and fumonisin production) (Picot et al. 2011). In addition, a relationship between resistance levels of maize and their 5-CQA content in immature kernels was demonstrated. Our results illustrated the ability of $F$. verticillioides to biotransform 5CQA with an efficiency that, in our experimental conditions, remains limited: more than $25 \%$ of 5-CQA present at the time of inoculation was retrieved after 21 days of incubation. The mechanism underlying 5-CQA-mediated reduction of fumonisin biosynthesis has not been yet elucidated. In the literature, multiple pieces of evidence suggest that oxidative stress is an enhancing factor for the biosynthesis of several mycotoxins (Picot et al. 2010; Reverberi et al. 2010). Consequently, the efficiency of phenolic acids in reducing toxin production was proposed to be possibly linked to their antioxidant properties (Kim et al. 2006; Ponts et al. 2011). Regarding fumonisin inhibition by 5-CQA and CA, we hypothesize that these two antioxidant compounds affect the mitochondrial respiration chain and, therefore, reduce the amount of acetyl-CoA originating from $\beta$-oxidation of short-chain fatty acids required in the early steps of fumonisin biosynthesis. An alternative hypothesis is that compounds with antioxidant activity such as 5-CQA and CA directly affect gene transcription or enzymatic activity involved in the fumonisin biosynthetic pathway. This pathway includes many oxidation steps that require high oxygen levels (Butchko et al. 2006). For example, antioxidants may inhibit redox enzymes such as P450 cytochrome monooxygenases, notably encoded by the FUM6 gene. Further studies have been undertaken to identify target genes for 5-CQA and CA.

Given that F. verticillioides can hydrolyze 5-CQA into CA and that $\mathrm{CA}$ was shown to be more efficient in inhibiting fumonisin biosynthesis, the question is whether inhibition is due to 5-CQA, 5-CQA-derived CA, or both. Moreover, the involvement of the metabolites hydroxychlorogenic, PA, and hydroxyCA (even though they reached very low levels in our experimental conditions) also needs to be investigated. In particular, eventual synergistic interactions that can enhance the inhibition of fumonisin production when all these phenolic compounds occur in mixture should be considered.

Altogether, these data provide evidence that 5-CQA may play a key role in maize resistance to fumonisin accumulation and could be a promising resistant biomarker. Such use of 5-CQA as a resistance biomarker requires validation, considering a wider

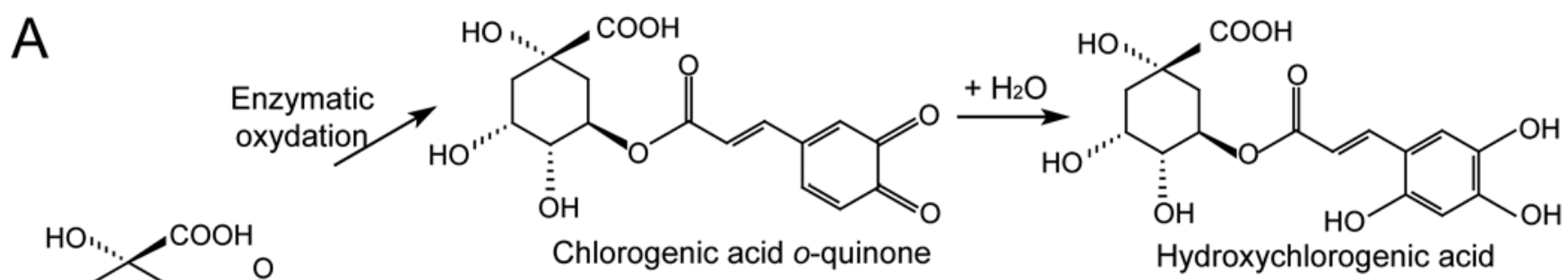<smiles>CC1(C)CC(OC(=O)/C=C/c2ccc(O)c(O)c2)[C@@H](O)[C@@H](O)C1</smiles>

Chlorogenic acid

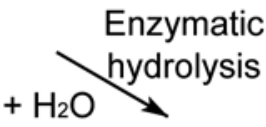<smiles>O=C(O)[C@]1(O)C[C@@H](O)[C@H](O)C[C@H]1O</smiles>

Quinic acid<smiles>O=C(O)/C=C/c1ccc(O)c(O)c1</smiles>

Caffeic acid
B<smiles>O=C(O)/C=C/c1ccc(O)c(O)c1</smiles>

Caffeic acid Enzymatic oxydation
and decarboxylation

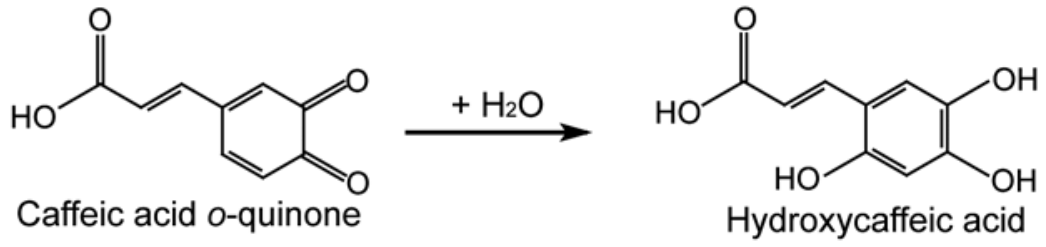

Hydroxycaffeic acid<smiles>O=C(OCc1ccccc1)Oc1ccc(C(=O)O)cc1O</smiles>

Fig. 5. Proposed pathways for aerobic biotransformation of A, chlorogenic acid and B, caffeic acid by Fusarium verticillioides Fv 63. 
set of genotypes. This validation has also to include seasonal monitoring of 5-CQA concentrations in a wide range of environments to ascertain the effect of phenology on the chemical composition of maize kernels harvested at the milk-dough stage.

\section{MATERIALS AND METHODS}

\section{Plant material.}

The maize inbred line used in the first part of our project (bioguided fractionation approach) is a genotype moderately susceptible to Fusarium ear rot, provided by BIOGEMMA Auvergne (Clermont-Ferrand, France). Untreated maize ears were harvested at 20 days after silking (silking was assigned when silk emergence could be observed in $50 \%$ of maize ears), frozen in liquid nitrogen, and stored at $-80^{\circ} \mathrm{C}$. Kernels were removed from lyophilized ears and ground to a fine powder before storage at $-20^{\circ} \mathrm{C}$.

To determine the relationship between resistance level to Fusarium ear rot and kernel composition in 5-CQA, healthy kernels from a set of 10 genotypes were used: 9 genotypes of the panel considered by Picot and associates (2013) in addition to the genotype selected for the identification of "antifumonisin" compounds. The 10 genotypes tested discriminate in three groups according to their levels of resistance to Fusarium ear rot: highly susceptible, susceptible, and moderately susceptible. This classification is based on symptom scores over several years of field experiments in France. The noninfected kernels were hand collected 20 days after silking.

\section{Fusarium strains.}

Ten strains of fumonisin-producing Fusarium spp. were used in this study: six F. verticillioides (Fv 62, Fv 63, Fv 64, Fv 368, $\mathrm{Fv} 424$, and Fv 444) and four F. proliferatum (Fp 58, Fp 367, Fp 381, and Fp 393). These strains were previously isolated from naturally infected maize kernels and belong to the MycSA collection (UR 1264 MycSA, INRA Bordeaux, France). Stock cultures were maintained at $4^{\circ} \mathrm{C}$ on potato dextrose agar (PDA) slants under mineral oil. When inoculum was required, the Fusarium strains were grown on PDA slants at $25^{\circ} \mathrm{C}$ in the dark for 7 days and spore suspensions were prepared by adding $6 \mathrm{ml}$ of sterile distilled water to the PDA slant, with gentle shaking.

\section{Medium and culture conditions.}

Liquid-culture experiments were performed in a glucose, amylopectin, yeast extract, and peptone (GAYEP) medium in 25-ml Erlenmeyer flasks (Picot et al. 2013). For each strain and condition, 10-ml aliquots of GAYEP, supplemented or not with maize crude extracts, with chromatographic fractions or standard bioactive compounds (Sigma-Aldrich, Lyon, France), were inoculated with $2 \times 10^{4}$ spores per milliliter. Flasks were then incubated in darkness at $25^{\circ} \mathrm{C}$ with shaking at $125 \mathrm{rpm}$ in a Multitron incubator (INFORS AG, Bottmingen, Switzerland) for 10 or 15 days. Additional incubation times were included for kinetic study $(2,4,7,10$, and 21 days). Following incubation, cultures were centrifuged at $3,000 \times g$ for $10 \mathrm{~min}$. Supernatants were stored at $-20^{\circ} \mathrm{C}$ until analysis. Fungal biomass was measured by weighing the mycelial pellet after $48 \mathrm{~h}$ of freeze-drying (Flexi-Dry, OErlikon Leybold, Germany). Lyophilized mycelia were stored at $-20^{\circ} \mathrm{C}$ until analysis. Cultures were done in triplicate. An appropriate control for each study was performed. It was verified that the initial $\mathrm{pH}$ of the culture medium was not affected by the supplementation and that final $\mathrm{pH}$ conditions did not vary between treatments.

\section{Maize kernel extracts.}

Ground maize (50 g) was suspended twice in $500 \mathrm{ml}$ of hexane for 30 min under agitation to extract nonpolar compounds.
After centrifugation $5 \mathrm{~min}$ at $3,000 \times g$, extracts were pooled, filtered through a $0.45 \mu \mathrm{m}$ porosity filter, concentrated in a rotavapor (BÜCHI Rotavapor R-200) at $30^{\circ} \mathrm{C}$ and stored until further use at $-20^{\circ} \mathrm{C}$.

Defatted maize was dried for $24 \mathrm{~h}$ at ambient temperature. Polar compounds were extracted from $15 \mathrm{~g}$ of defatted maize after $3 \mathrm{~h}$ of agitation with $240 \mathrm{ml}$ of water. Semipolar compounds were extracted from $15 \mathrm{~g}$ of maize with $240 \mathrm{ml}$ of methanol/water $(70: 30, \mathrm{vol} / \mathrm{vol})$ acidified with $0.1 \%$ acetic acid and $12 \mathrm{~h}$ of agitation. Water and organic extracts were filtered through $0.45-\mu \mathrm{m}$ porosity filters, concentrated in a rotavapor (BÜCHI Rotavapor R-200) at $40^{\circ} \mathrm{C}$, and lyophilized (Flexi-Dry). Nonpolar, semipolar, and polar extracts were tested at $500 \mathrm{mg}$ of dry matter per liter on fungal growth and fumonisin accumulation by $F$. verticillioides Fv 63 .

Methanol/water extract was produced as many times as necessary, 10-fold concentrated in a rotavapor (BÜCHI Rotavapor $\mathrm{R}-200$ ) at $40^{\circ} \mathrm{C}$, and stored at $-20^{\circ} \mathrm{C}$ before further fractionation.

\section{Fractionation of active maize extract.}

The 10-fold concentrated maize methanol/water extract ( 5 $\mathrm{ml})$ was filtered through a $0.45-\mu \mathrm{m}$-pore-size Millipore filter and loaded onto a Toyopearl Fractogel HW-40S (Interchim, San Pedro. CA, U.S.A.) column (26 by $320 \mathrm{~mm}$ ) using an ÄKTA prime chromatographic system (Amersham Biosciences, Piscataway, NJ, U.S.A.). The column was equilibrated and eluted first with $600 \mathrm{ml}$ of water, then with $800 \mathrm{ml}$ of methanol. The elution rate was $60 \mathrm{ml} / \mathrm{h}$. Absorbances at 230 , 280, and $320 \mathrm{~nm}$ were determined for each 7-ml collected tube. According to the absorbance monitoring, tubes were pooled (F1, F2, F3, and F4) concentrated, and lyophilized. Each F1, F2, F3, and F4 fraction (500 mg of dry matter per liter) was dissolved in GAYEP medium to evaluate antifungal and fumonisin inhibitory activities. Negative controls were performed with water (for fractions F1 and F2) and methanol (for fractions F3 and F4) and collected at the beginning and the end of the washing procedure, following the above protocol. It was previously checked that initial $\mathrm{pH}$ of the culture medium was not affected by supplementation with these fractions compared with the controls. Cultures were done in triplicate.

\section{LC/DAD.}

LC/DAD analyses were performed using an 1100 Series high-performance liquid chromatography (HPLC) system (Agilent, Massy, France) equipped with an autosampler system, an Agilent photodiode array detector, and ChemStation chromatography manager software (Agilent). Chromatographic separation was achieved on a Kinetex XB-C18 $100 \AA$ column ( 150 by $4.60 \mathrm{~mm}, 2.6 \mu$ ) (Phenomenex, Le Pecq, France) protected with a guard column of the same material and maintained at $45^{\circ} \mathrm{C}$. The mobile phase consisted of $0.2 \%$ formic acid in water ( $\mathrm{vol} / \mathrm{vol}$ ) (solvent $\mathrm{A}$ ) and $0.2 \%$ formic acid in acetonitrile ( $\mathrm{vol} / \mathrm{vol}$ ) (solvent B). The gradient elution was as follows: 5 to $20 \%$ B for $10 \mathrm{~min}, 20$ to $95 \%$ B for $2 \mathrm{~min}, 95 \% \mathrm{~B}$ for $5 \mathrm{~min}, 95$ to $5 \% \mathrm{~B}$ for $2 \mathrm{~min}$, and $5 \%$ for $10 \mathrm{~min}$ postrun reconditioning. The injection volume was $5 \mu \mathrm{l}$. The flow rate was kept at $1 \mathrm{ml} / \mathrm{min}$. The UV-visible spectra were recorded from 190 to $500 \mathrm{~nm}$ and peak areas were measured at 260 and $320 \mathrm{~nm}$, according to the molecule. Quantification was performed by external calibrations with standard solutions prepared from commercial pure powders of 5-CQA, CA, PA, and benzoxazolin-2-one (BOA) (Sigma-Aldrich). The concentrations of active compounds in chromatographic fractions were expressed in milligrams per gram of lyophilized fraction. For in vitro supplementation experiments, concentrations retrieved in supernatants or mycelia were expressed in micromoles. Hydroxychlorogenic acid and hydroxyCA were quantified at 
$320 \mathrm{~nm}$ and peak areas were converted in micromoles of equivalent 5-CQA and CA, respectively. FQA and $p$-CoQA were quantified at $320 \mathrm{~nm}$ and peak areas were converted in milligram equivalent of 5-CQA per gram of analyzed fraction. HMBOA-Glc and DIMBOA-Glc were quantified at $260 \mathrm{~nm}$ and peak areas were converted in milligram equivalent BOA per gram of analyzed fraction.

\section{LC-MS analysis.}

MS analysis and fragmentation experiments were performed on a QTrap 2000 LC/MS/MS system (Applied Biosystems, Les Ulis, France) equipped with an ESI source and an 1100 Series HPLC system (Agilent), and on a micro-quadrupole time-of-flight LC/MS system (Bruker, Leipzig, Germany) equipped with an ESI source and an Ultimate 3000 HPLC system (Dionex, Courtaboeuf, France). Chromatographic conditions were the same as described above. The flow rate was kept at $1 \mathrm{ml} / \mathrm{min}$ and was split after UV-visible detection so that $0.25 \mathrm{ml} / \mathrm{min}$ went to the electrospray source. Compound structures were resolved by LC-ESI/MS according to the method described previously (Boutigny et al. 2010).

\section{Extraction and analysis of fumonisins and 5-CQA.}

Fumonisins were extracted from culture media and analyzed by LC fluorescence detector, as described previously (Picot et al. 2013). The sum of FB1, FB2, and FB3 was used to quantitate the amount of fumonisins and results were converted to micrograms per gram of dry fungal biomass.

5-CQA was extracted from lyophilized maize powders and quantified by HPLC-DAD, as described previously (AtanasovaPenichon et al. 2012). Concentrations of 5-CQA in maize were expressed in micrograms per gram of dry maize powder.

\section{Extraction and analysis of products}

\section{issued from 5-CQA and CA biotransformation.}

Products resulting from biotransformation of 5-CQA and CA in supernatants were directly analyzed by LC/DAD and LC-MS, as described above, after filtration of supernatant on a $0.45-\mu \mathrm{m}$ filter.

Products resulting from biotransformation of 5-CQA and $\mathrm{CA}$ in mycelia were extracted by agitating $0.05 \mathrm{~g}$ of lyophilized and ground mycelium with $1 \mathrm{ml}$ of methanol/water (70:30, $\mathrm{vol} / \mathrm{vol}$ ), acidified with $0.1 \%$ formic acid for $1 \mathrm{~h}$. After centrifugation (at 7,000 $\times g$ for $5 \mathrm{~min}$ ), supernatant was filtered on a $0.45-\mu \mathrm{m}$ filter and analyzed by LC/DAD and LC-MS, as described above.

\section{Expression of results and statistical analyses.}

Results for fumonisin production were reported as mean values \pm standard deviation of three biological replications. Values were compared at the 5\% significance level using a standard Student's $t$ test (control versus treated).

Statistical analysis for 5-CQA was performed using XLSTAT 2008 software (Addinsoft, Rennes, France) using analysis of variance. Differences between three groups of genotypes were determinate with a multiple comparisons test using the Duncan method. Levels of significance was set at $\alpha=0.05$.

\section{ACKNOWLEDGMENTS}

We thank BIOGEMMA Auvergne (Clermont-Ferrand, France) for providing the maize inbred line used throughout the study and V. C. Huskins for his attentive proofreading. This work was partly supported by Agence Nationale de la Recherche (ANR) through the Project ANR 'MoniMaize' GPLA-07-043C. One part of the research leading to these results has received funding from the European Union Seventh Framework Programme (FP7/2007-2013) under the grant agreement number 265865 PURE.

\section{LITERATURE CITED}

Ahmad, S., Veyrat, N., Gordon-Weeks, R., Zhang, Y. H., Martin, J., Smart, L., Glauser, G., Erb, M., Flors, V., Frey, M., and Ton, J. 2011. Benzoxazinoid metabolites regulate innate immunity against aphids and fungi in maize. Plant Physiol. 157:317-327.

Alonso-Salces, R. M., Guillou, C., and Berrueta, L. A. 2009. Liquid chromatography coupled with ultraviolet absorbance detection, electrospray ionization, collision-induced dissociation and tandem mass spectrometry on a triple quadrupole for the on-line characterization of polyphenols and methylxanthines in green coffee beans. Rapid Commun. Mass Spectrom. 23:363-383.

Assabgui, R., Reid, L., Hamilton, R., and Arnason, J. 1993. Phenolics in maize genotypes differing in susceptibility to Gibberella stalk rot (Fusarium graminearum Schwabe). Phytopathology 83:949-953.

Asther, M., Estrada Alvarado, M. I., Haon, M., Navarro, D., Asther, M., Lesage-Meessen, L., and Record, E. 2005. Purification and characterization of a chlorogenic acid hydrolase from Aspergillus niger catalysing the hydrolysis of chlorogenic acid. J. Biotechnol. 115:47-56

Atanasova-Penichon, V., Pons, S., Pinson-Gadais, L., Picot, A., Marchegay, G., Bonnin-Verdal, M.-N., Ducos, C., Barreau, C., Roucolle, J., Sehabiague, P., Carolo, P., and Richard-Forget, F. 2012. Chlorogenic acid and maize ear rot resistance: A dynamic study investigating Fusarium graminearum development, deoxynivalenol production, and phenolic acid accumulation. Mol. Plant-Microbe Interact. 25:16051616.

Bakan, B., Bily, A. C., Melcion, D., Cahagnier, B., Regnault-Roger, C., Philogene, B. J. R., and Richard-Molard, D. 2003. Possible role of plant phenolics in the production of Trichothecenes by Fusarium graminearum strains on different fractions of maize kernels. J. Agric. Food Chem. 51:2826-2831.

Balcerzak, M., Harris, L. J., Subramaniam, R., and Ouellet, T. 2012. The feruloyl esterase gene family of Fusarium graminearum is differentially regulated by aromatic compounds and hosts. Fungal Biol. 116:478-488.

Barberis, C., Astoreca, A., Fernandez-Juri, M. G., Dalcero, A. M., and Magnoli, C. 2010. Effect of antioxidant mixtures on growth and ochratoxin A production of Aspergillus section Nigri species under different water activity conditions on peanut meal extract agar. Toxins 2:13991413.

Beekrum, S., Govinden, R., Padayachee, T., and Odhav, B. 2003. Naturally occurring phenols: A detoxification strategy for fumonisin B1. Food Addit. Contam. 20:490-493.

Benoit, I., Asther, M., Bourne, Y., Navarro, D., Canaan, S., LesageMeessen, L., Herweijer, M., Coutinho, P. M., Asther, M., and Record, E. 2007. Gene overexpression and biochemical characterization of the biotechnologically relevant chlorogenic acid hydrolase from Aspergillus niger. Appl. Environ. Microbiol. 73:5624-5632.

Berthiller, F., Dall'asta, C., Corradini, R., Marchelli R., Sulyok, M., Krska, R., Adam, G., and Schuhmacher, R. 2009. Occurrence of deoxynivalenol and its 3-D-glucoside in wheat and maize. Food Addit. Contam. Part A Chem. Anal. Control Expo. Risk Assess. 26:507-511.

Bollina, V., Kumaraswamy, G. K., Kushalappa, A. C., Choo, T. M., Dion, Y., Rioux, S., Faubert, D., and Hamzehzarghani, H. 2010. Mass spectrometry-based metabolomics application to identify quantitative resistance-related metabolites in barley against Fusarium head blight. Mol. Plant Pathol. 11:769-782.

Bonnington, L. S., Barcelò, D., and Knepper, T. P. 2003. Utilisation of electrospray time-of-flight mass spectrometry for solving complex fragmentation patterns: Application to benzoxazinone derivatives. J. Mass. Spectrom. 38:1054-1066.

Boutigny, A.-L., Richard-Forget, F., and Barreau, C. 2008. Natural mechanisms for cereal resistance to the accumulation of Fusarium trichothecenes. Eur. J. Plant Pathol. 121:411-423.

Boutigny, A.-L., Barreau, C., Atanasova-Penichon, V., Verdal-Bonnin, M.N., Pinson-Gadais., L., and Richard-Forget, F. 2009. Ferulic acid, an efficient inhibitor of type B trichothecene biosynthesis and Tri gene expression in Fusarium liquid cultures. Mycol. Res. 113:746-753.

Boutigny, A.-L., Atanasova-Penichon, V., Benet, M., Barreau, C., and Richard-Forget, F. 2010. Natural phenolic acids from wheat bran inhibit Fusarium culmorum trichothecene biosynthesis in vitro by repressing Tri gene expression. Eur. J. Plant Pathol. 127:275-286.

Brown, D. W., Butchko, R. A. E., Busman, M., and Proctor, R. H. 2012. Identification of gene clusters associated with fusaric acid, fusarin, and perithecial pigment production in Fusarium verticillioides. Fungal Genet. Biol. 49:521-532.

Butchko, R. A. E., Plattner, R. D., and Proctor, R. H. 2006. Deletion analysis of FUM genes involved in tricarballylic ester formation during fumonisin biosynthesis. J. Agric. Food Chem. 54:9398-9404.

Cambier, V., Hance, T., and de Hoffmann, E. 1999. Non-injured maize contains several 1,4-benzoxazin-3-one related compounds but only as 
glucoconjugates. Phytochem. Anal. 10:119-126.

Campos-Bermudez, V. A., Fauguel, C. M., Tronconi, M. A., Casati, P., Presello, D. A., and Andreo, C. S. 2013. Transcriptional and metabolic changes associated to the infection by Fusarium verticillioides in maize inbreds with contrasting ear rot resistance. PLoS One 8:e61580.

Chipley, J. R., and Uraih, N. 1980. Inhibition of Aspergillus growth and aflatoxin release by derivatives of benzoic-acid. Appl. Environ. Microbiol. 40:352-357.

Clifford, M. N., Johnston, K. L., Knight, S., and Kuhnert, N. 2003. Hierarchical scheme for LC-MS ${ }^{\mathrm{n}}$ identification of chlorogenic acids. J. Agric. Food Chem. 51:2900-2911.

Clifford, M. N., Kirkpatrick, J., Kuhnert, N., Roozendaal, H., and Salgado, P. R. 2008. LC-MSn analysis of the cis isomers of chlorogenic acids. Food Chem. 106:379-385.

Dambolena, J. S., Lopez, A. G., Canepa, M. C., Theumer, M. G., Zygadlo, J. A., and Rubinstein, H. R. 2008. Inhibitory effect of cyclic terpenes (limonene, menthol, menthone and thymol) on Fusarium verticillioides MRC 826 growth and fumonisin B1 biosynthesis. Toxicon 51:37-44.

Dixon, D. P., Sellars, J. D., Kenwright, A. M., and Steel, P. G. 2012. The maize benzoxazinone DIMBOA reacts with glutathione and other thiols to form spirocyclic adducts. Phytochemistry 77:171-178.

Edwards, S. G. 2004. Influence of agricultural practices on Fusarium infection of cereals and subsequent contamination of grain by trichothecene mycotoxins. Toxicol. Lett. 153:29-35.

Gelderblom, W. C. A., Rheeder, J. P., Leggott, N., Stockenstrom, S., Humphreys, J., Shephard, G. S., and Marasas, W. F. O. 2004. Fumonisin contamination of a corn sample associated with the induction of hepatocarcinogenesis in rats-role of dietary deficiencies. Food Chem. Toxicol. 42:471-479.

Glenn, A. E., Hinton, D. M., Yates, I. E., and Bacon, C. W. 2001. Detoxification of corn antimicrobial compounds as the basis for isolating Fusarium verticillioides and some other Fusarium species from corn. Appl. Environ. Microbiol. 67:2973-2981.

Glenn, A. E., Gold, S. E., and Bacon, C. W. 2002. Fdb1 and Fdb2, Fusarium verticillioides loci necessary for detoxification of preformed antimicrobials from corn. Mol. Plant-Microbe Interact. 15:91-101.

Guiraud, P., Steiman, R., Seiglemurandi, F., and Benoitguyod, J. L. 1995. Comparison of the toxicity of various lignin-related phenolic-compounds toward selected fungi perfecti and fungi imperfecti. Ecotoxicol. Environ. Safe. 32:29-33.

Humpf, H. U., and Voss, K. A. 2004. Effects of thermal food processing on the chemical structure and toxicity of fumonisin mycotoxins. Mol. Nutr. Food Res. 48:255-269.

Kader, F., Irmouli, M., Nicolas, J. P., and Metche, M. 2001. Proposed mechanism for the degradation of pelargonidin 3-glucoside by caffeic acid o-quinone. Food Chem. 75:139-144

Kim, J. H., Mahoney, N., Chan, K. L., Molyneux, R. J., and Campbell, B. C. 2006. Controlling food-contaminating fungi by targeting their antioxidative stress-response system with natural phenolic compounds. Appl. Microbiol. Biotechnol. 70:735-739.

Kumaraswamy, G., Bollina, V., Kushalappa, A., Choo, T., Dion, Y., Rioux S., Mamer, O., and Faubert, D. 2011. Metabolomics technology to phenotype resistance in barley against Gibberella zeae. Eur. J. Plant Pathol. 130:29-43.

Lattanzio, V., Lattanzio, V., and Cardinali, A. 2006. Role of phenolics in the resistance mechanisms of plants against fungal pathogens and insects. Phytochemistry 2:23-67.

Leiss, K. A., Maltese, F., Choi, Y. H., Verpoorte, R., and Klinkhamer, P. G.L. 2009. Identification of chlorogenic acid as a resistance factor for thrips in chrysanthemum. Plant Physiol. 150:1567-1575.

Mahoney, N., and Molyneux, R. J. 2004. Phytochemical inhibition of aflatoxigenicity in Aspergillus flavus by constituents of walnut (Juglans regia). J. Agric. Food Chem. 52:1882-1889.

Miller, J. D., Fielder, D. A., Dowd, P. F., Norton, R. A., and Collins, F. W. 1996. Isolation of 4-acetyl-benzoxazolin-2-one (4-ABOA) and diferuloylputrescine from an extract of Gibberella ear rot-resistant corn that blocks mycotoxin biosynthesis, and the insect toxicity of 4-ABOA and related compounds. Biochem. Syst. Ecol. 24:647-658.
Niemeyer, H. M. 2009. Hydroxamic acids derived from 2-Hydroxy-2H1,4-benzoxazin-3(4H)-one: Key defense chemicals of cereals. J. Agric. Food Chem. 57:1677-1696.

Norton, R. A. 1999. Inhibition of aflatoxin B-1 biosynthesis in Aspergillus flavus by anthocyanidins and related flavonoids. J. Agric. Food Chem. 47:1230-1235

Paster, N., Juven, B. J., and Harshemesh, H. 1988. Antimicrobial activity and inhibition of aflatoxin-B1 formation by olive plant-tissue constituents. J. Appl. Bacteriol. 64:293-297.

Picot, A., Barreau, C., Pinson-Gadais, L., Caron, D., Lannou, C., and Richard-Forget, F. 2010. Factors of the Fusarium verticillioides-maize environment modulating fumonisin production. Crit. Rev. Microbiol. $36: 221-231$.

Picot, A., Barreau, C., Pinson-Gadais, L., Piraux, F., Caron, D., Lannou, C., and Richard-Forget, F. 2011. The dent stage of maize kernels is the most conducive for fumonisin biosynthesis under field conditions. Appl. Environ. Microbiol. 77:8382-8390.

Picot, A., Atanasova-Pénichon, V., Pons, S., Marchegay, G., Barreau, C., Pinson-Gadais, L., Roucolle, J., Daveau, F., Caron, D., and RichardForget, F. 2013. Maize kernel antioxidants and their potential involvement in Fusarium ear rot resistance. J. Agric. Food Chem. 61:33893395

Ponts, N., Pinson-Gadais, L., Boutigny, A.-L., Barreau, C., and RichardForget, F. 2011. Cinnamic-derived acids significantly affect Fusarium graminearum growth and in vitro synthesis of type B trichothecenes. Phytopathology 101:929-934.

Reid, L. M., Arnason, J. T., Nozzolillo, C., and Hamilton, R. I. 1991. Laboratory and field-resistance to the European corn-borer in maize germplasm. Crop Sci. 31:1496-1502.

Reverberi, M., Ricelli, A., Zjalic, S., Fabbri, A. A., and Fanelli, C. 2010. Natural functions of mycotoxins and control of their biosynthesis in fungi. Appl. Microbiol. Biotechnol. 87:899-911.

Richard-Forget, F. C., Rouet-Mayer, M. A., Goupy, P. M., Philippon, J., and Nicolas, J. J. 1992. Oxidation of chlorogenic acid, catechins, and 4methylcatechol in model solutions by apple polyphenol oxidase. J. Agric. Food Chem. 40:2114-2122.

Samapundo, S., De Meulenaer, B., Osei-Nimoh, D., Lamboni, Y., Debevere, J., and Devlieghere, F. 2007. Can phenolic compounds be used for the protection of corn from fungal invasion and mycotoxin contamination during storage? Food Microbiol. 24:465-473.

Sanchez-Rangel, D., SanJuan-Badillo, A., and Plasencia, J. 2005. Fumonisin production by Fusarium verticillioides strains isolated from maize in Mexico and development of a polymerase chain reaction to detect potential toxigenic strains in grains. J. Agric. Food Chem. 53:8565-8571.

Shin, H. D., and Chen, R. R. Z. 2006. Production and characterization of a type B feruloyl esterase from Fusarium proliferatum NRRL 26517. Enzyme Microb. Technol. 38:478-485.

Soltoft, M., Jorgensen, L. N., Svensmark, B., and Fomsgaard, I. S. 2008 Benzoxazinoid concentrations show correlation with Fusarium Head Blight resistance in Danish wheat varieties. Biochem. Syst. Ecol. 36:245-259.

Torres-Mancera, M. T., Baqueiro-Pena, I., Figueroa-Montero, A., RodriguezSerrano, G., Gonzalez-Zamora, E., Favela-Torres, E., and SaucedoCastaneda, G. 2013. Biotransformation and improved enzymatic extraction of chlorogenic acid from coffee pulp by filamentous fungi. Biotechnol. Prog. 29:337-345.

Villarino, M., Sandin-Espana, P., Melgarejo, P., and De Cal, A. 2011. High chlorogenic and neochlorogenic acid levels in immature peaches reduce Monilinia laxa infection by interfering with fungal melanin biosynthesis. J. Agric. Food Chem. 59:3205-3213.

Voss, K. A., Howard, P. C., Riley, R. T., Sharma, R. P., Bucci, T. J., and Lorentzen, R. J. 2002. Carcinogenicity and mechanism of action of fumonisin B-1: A mycotoxin produced by Fusarium moniliforme $(=F$ verticillioides). Cancer Detect. Prev. 26:1-9.

Zheng, Y. Q., Zhao, Y., Dong, F. S., Yao, J. R., and Hurle, K. 2005. Relationship of DIMBOA content in wheat seedlings and its resistance to plant pathogens. Allelopathy J. 15:137-143. 